2021-01

\title{
Is there an association between drug use and oral health conditions?
}

\author{
Paisi, Martha
}

http://hdl.handle.net/10026.1/18297

10.1038/s41432-021-0158-2

Evidence-Based Dentistry

Springer Science and Business Media LLC

All content in PEARL is protected by copyright law. Author manuscripts are made available in accordance with publisher policies. Please cite only the published version using the details provided on the item record or document. In the absence of an open licence (e.g. Creative Commons), permissions for further reuse of content should be sought from the publisher or author. 


\section{Summary Review / DENTAL PUBLIC HEALTH}

\section{Title/Question}

Is there an association between drug use and oral health conditions?

\section{Authors}

Martha Paisi, ${ }^{1,2}$ Robert Witton, ${ }^{1,2}$ Anastasios Plessas ${ }^{1}$

${ }^{1}$ Peninsula Dental School, University of Plymouth, Plymouth, United Kingdom.

martha.paisi@plymouth.ac.uk robert.witton@plymouth.ac.uk anastasios.plessas@plymouth.ac.uk

${ }^{2}$ Peninsula Dental Social Enterprise, University of Plymouth, Plymouth, United Kingdom. robert.witton@plymouth.ac.uk 


\section{A Commentary on}

Yazdanian M, Armoon B, Noroozi A, Mohammadi R, Bayat AH, Ahounbar E, Higgs

P, Nasab HS, Bayani A, Hemmat M. Dental caries and periodontal disease among people who use drugs: a systematic review and meta-analysis. BMC Oral Health. 2020 Feb 10;20(1):44. doi: 10.1186/s12903-020-1010-3.

\section{Data sources}

Databases searched included PsycINFO, PubMed, SciELO, Scopus, and Web of Science.

\section{Study selection}

Cross-sectional, longitudinal and retrospective studies that compared caries, periodontal disease or tooth loss in people who use drugs as compared to those who do not. Studies that included psychiatric populations and alcohol or tobacco users were not included in the review. Qualitative studies, in vitro investigations, animal studies, reviews, case reports and series, letters to editor, and conference abstracts were also excluded. The authors included only English studies published before July $1^{\text {st }}$ 2019. Two independent reviewers screened the papers on title and abstract and then full text. In case of disagreements, these were discussed between the two reviewers and a third one was consulted if needed. 


\section{Data extraction and synthesis}

Two reviewers extracted the data and contacted the primary authors for necessary clarifications, if needed. The unweighted kappa was applied to examine inter-examiner agreement. The Joanna Briggs Institute Critical Appraisal Checklist for observational studies was used to critically appraise the studies. The study selection results were presented through a flow chart. For the meta-analysis, the authors considered adjusted data. In some cases, crude estimates were used. Hetereogeneity was estimated using the $\mathrm{I}^{2}$ statistic. The 'meta' package was used for the meta-analysis.

\section{Results}

Ten studies were included in the meta-analysis. Drug use was associated with higher risk of periodontal disease (OR 1.44; 95\% $\mathrm{Cl}$ 0.8-2.6) and higher DMFT index (OR $4.11 ; 95 \% \mathrm{Cl} 2.07-8.15)$.

\section{Conclusions}

The review showed high risk of periodontal disease and caries among people who use drugs. The authors concluded that this association may be explained by irregular toothbrushing and long history of drug use. It is important to develop programs that aim to improve oral hygiene practices among people who use drugs.

GRADE Rating: Low (two out of four EBD stars) 


\section{Commentary}

Dental health problems are among the most prevalent comorbidities associated with long-term drug use. ${ }^{1,2}$ Chronic drug use has been associated with higher risk for dental caries, periodontal disease, xerostomia, tooth wear, and tooth loss. ${ }^{2,3}$ Dental health complications may be attributed to a) the direct impact of the chemical qualities of the illicit substances on oral tissues (mucosa, gingivae and teeth) ${ }^{4,5}$ and on saliva production; ${ }^{6,7}$ and b) the user's associated health risk behaviours (i.e. poor oral hygiene and high consumption of refined carbohydrates). ${ }^{4,8-9}$

The aim of this systematic review and meta-analysis was to examine the association between drug use and oral health. In terms of oral health conditions, the authors focused on periodontal disease and dental caries. Outcomes in terms of illicit drugs included methamphetamines, cocaine, heroin, opiates, crack and cannabis.

Following de-duplication and screening 10 papers were included in the review. The age or gender of the participants was not provided, nor the duration of drug use. The sample sizes of the included studies ranged from 18 to 1015 . Five of the studies were cross-sectional, four were case-control and another one was a cohort study. The drugs used by participants (some refer to the same study) included methamphetamine ( 3 studies), cannabis (2 studies), heroin (2 studies), opiate (2 studies), amphetamines (1 study), opioid (1 study), marihuana (1 study) and illicit drugs (1 study). Pooled estimates demonstrated that drug use was associated with higher risk of periodontal disease (OR 1.44; 95\% Cl 0.8-2.6) and dental caries (DMFT) (OR 4.11; 95\% Cl 2.078.15). 
The protocol was not registered a priory. The PRISMA guidelines were followed for the conduct and reporting of the review. The inclusion and exclusion criteria could have been defined using the PICO framework. The decisions for some of the exclusion criteria lack clear justification. For example, the authors seem to have excluded studies which recruited people who 'use alcohol or smoke tobacco', although it has been shown that a considerable proportion of use drugs also consume alcohol and/or tobacco. ${ }^{4,10}$ Furthermore, studies involving people experiencing homelessness, among whom drug use is known to be prevalent, were excluded. ${ }^{11}$ The authors searched only online databases and only included studies in English language, and therefore there is an increased risk of publication bias. However, Begg's test found no evidence of publication bias for periodontal disease or caries. The search strategies used are clearly presented. Although a critical appraisal was conducted, the authors did not specify how this may have influenced interpretation of results. The findings of this review and meta-analysis are limited by the fact that the majority of studies were of cross- sectional design. Hence, causation was difficult to infer. Very importantly, the $1^{2}$ statistic indicated high heterogeneity between studies and as a result there is high uncertainty in the effect estimates. Therefore, the results and conclusions of this systematic review should be interpreted with caution.

Consistently with another systematic review and meta-analysis, ${ }^{4}$ the present review showed that drug use is associated with higher risk of periodontal disease and dental caries. In addition to the direct impact of drugs on oral health tissues, this could be suggestive of poor oral hygiene and low utilisation of dental services among people 
who use illicit substances. ${ }^{4,12}$ Considering the negative impact of long-term drug use on oral health, targeted interventions to improve oral health habits and access to dental services for people who use drugs are warranted.

\section{Practice Points}

- People who use drugs have higher risk to develop caries and periodontal disease than their non-drug using peers.

- Dental health professionals should screen for substance use as part of a standard medical history in their practices, and provide enhanced prevention for patients who are engaged in substance misuse.

- Dental screening in drug treatment services could enable early detection of dental health problems and facilitate early access to dental care services.

- Oral health promotion activities should be integrated into drug treatment services delivery. 


\section{References}

1. Metsch LR, Crandall L, Wohler-Torres B, Miles CC, Chitwood DD, McCoy CB. Met and unmet need for dental services among active drug users in Miami, Florida. J Behav Health Serv Res. 2002;29(2):176-188.

2. Shekarchizadeh H, Khami MR, Mohebbi SZ, Ekhtiari H, Virtanen JI. Oral Health of Drug Abusers: A Review of Health Effects and Care. Iran J Public Health. 2013;42(9):929-940.

3. Shetty V, Harrell L, Clague J, Murphy DA, Dye BA, Belin TR. Methamphetamine Users Have Increased Dental Disease: A Propensity Score Analysis. J Dent Res. 2016;95(7):814-821.

4. Baghaie H, Kisely S, Forbes M, Sawyer E, Siskind DJ. A systematic review and meta-analysis of the association between poor oral health and substance abuse. Addiction. 2017;112(5):765-779.

5. Mallatt ME. Meth mouth: a national scourge. J Indiana Dent Assoc. 2005;84(3):28-9.

6. Saini TS, Edwards PC, Kimmes NS, Carroll LR, Shaner JW, Dowd FJ, Saini T, Kimmes NS. Etiology of xerostomia and dental caries among methamphetamine abusers. Oral Health Prev Dentistry. 2005;3(3):189-95.

7. Joshi S, Ashley M. Cannabis: a joint problem for patients and the dental profession. Br Dent J. 2016; 220: 597-601.

8. Murphy DA, Harrell L, Fintzy R, Vitero S, Gutierrez A, Shetty V. Soda Consumption Among Methamphetamine Users in the USA: Impact on Oral Health. Oral Health Prev Dent. 2016;14(3):227-234.

9. Smit DA, Naidoo S. Oral health effects, brushing habits and management of methamphetamine users for the general dental practitioner. $\mathrm{Br}$ Dent $\mathrm{J}$. 2015. 218(9):531-536.

10. Kapusta ND, Ramskogler K, Hertling I, Schmid R, Dvorak A, Walter H, Lesch OM. (2006) Epidemiology of substance use in a representative sample of 18year-old males. Alcohol Alcohol. Mar-Apr;41(2):188-92.

11. Groundswell. Healthy Mouths: A peer-led health audit on the oral health of people experiencing homelessness. London: Groundswell; 2017.

12. Charnock S, Owen S, Brookes V, Williams M (2004). A community based programme to improve access to dental services for drug users. $\mathrm{Br}$ Dent $\mathrm{J}$. 2004;196(7):385-8. 